\title{
Collagen triple helix repeat containing 1 promotes endometrial cancer cell migration by activating the focal adhesion kinase signaling pathway
}

\author{
XIANG HU* ${ }^{*}$ YIDING BIAN* ${ }^{*}$ XIAOLI WEN, MENGFEI WANG, YIRAN LI and XIAOPING WAN \\ Department of Gynecology, Shanghai First Maternity and Infant Hospital, \\ Tongji University School of Medicine, Shanghai 200040, P.R. China
}

Received August 27, 2019; Accepted April 24, 2020

DOI: $10.3892 /$ etm.2020.8833

\begin{abstract}
Metastasis of endometrial cancer (EC) is known to be the major cause of relapse and death of patients. However, the mechanisms of this process are not well understood. Focal adhesion kinase (FAK) is known for its essential role in integrin signaling and is highly expressed in many human tumors. FAK also plays important roles in a variety of cellular processes. Collagen triple helix repeat containing 1 (CTHRC1) is a secreted protein that has been detected in many human solid cancers. In the present study, CTHRC1 was found to be highly expressed in EC tissues when compared with normal tissues. In addition, overexpression of CTHRC1 promoted the migration of EC cells in vitro. Recombinant CTHRC1 protein also promoted the migration of EC cells in vitro. The results of the present study suggested that CTHRC1 mediated EC cell migration via the FAK signaling pathway. Taken together, these data indicated that CTHRC1 and the FAK signaling pathway may be potential therapeutic targets for EC metastasis treatment.
\end{abstract}

\section{Introduction}

Endometrial cancer (EC) is the most frequently occurring malignant gynecological cancer (1). Globally, it accounts for approximately $5 \%$ of total cancer cases with a mortality rate of over $2 \%$ in women. EC can be grossly divided into two groups. The majority of cases of EC are type I, estrogen and progesterone receptor-positive cancers with chronic

Correspondence to: Dr Xiaoping Wan, Department of Gynecology, Shanghai First Maternity and Infant Hospital, Tongji University School of Medicine, 2699 West Gaoke Road, Pudong, Shanghai 200040, P.R. China

E-mail: wanxiaoping@tongji.edu.cn

*Contributed equally

Key words: collagen triple helix repeat containing 1, focal adhesion kinase, endometrial cancer, migration, mechanism estrogen exposure and insufficient opposing progesterone exposure $(2,3)$. In contrast, poorly differentiated type II ECs are primarily uterine serous carcinomas and are correlated with poor prognoses $(4,5)$. Although numerous patients suffering from EC in a low-grade or early-stage can have a good prognosis, the prognosis of metastatic or late-stage EC is still poor $(6,7)$. Therefore, valid therapies for advanced EC are needed.

Metastasis is an essential process in the progression of cancer and can be divided into two steps. First, the cancer cells translocate to a distant organ. The translocated cells then transform into a metastasis at the organ $(8,9)$. In EC, the metastatic process relies on alterations in the extracellular matrix (ECM) and cell-cell adhesion $(10,11)$. A potential signaling event in the metastasis of EC is the overexpression of focal adhesion kinase (FAK) (12). FAK is known for its essential role in integrin signaling and is overexpressed in a number of human tumors. It is also essential in a variety of cell processes such as growth, survival, invasion and migration (13-15). Furthermore, it has been shown that the migration of cancer cells can be blocked after inhibiting activation of the FAK signaling pathway $(16,17)$.

It has been reported that collagen triple helix repeat containing 1 (CTHRC1) plays an important role in vascular remodeling, morphogenesis and bone formation (18-20). Furthermore, recent studies have shown that CTHRC1 can be detected in human solid cancers, such as lung, breast, thyroid, pancreatic and ovarian cancers, and could reduce collagen matrix deposition (21-25). Although it has been reported that CTHRC1 is overexpressed in a number of human solid cancers, the molecular mechanism of CTHRC1 action in EC cells is unclear. It has been reported that CTHRC1 may promote the migration of pancreatic cancer cells via activation of the FAK signaling pathway (26). A previous study indicated that CTHRC1 is overexpressed in EC (27). However, the detailed mechanisms of CTHRC1 in EC remain unknown. Therefore, the goal of the current research was to explore the role of CTHRC1 in EC.

To the best of our knowledge, the present study is the first to indicate that CTHRC1 may promote the migration of EC cells. CTHRC1 was seen to be highly expressed in EC tissues. In addition, overexpression of CTHRC1 promoted the 
migration of EC cells in vitro. Recombinant CTHRC1 protein also promoted migration of EC cells. The results of the present study indicated that CTHRC1 mediated the migration of EC cells via the FAK signaling pathway.

\section{Materials and methods}

Ethics. The present study was approved by the Human Investigation Ethics Committee of the Shanghai First Maternity and Infant Hospital (Shanghai, China). Samples were collected from patients after receiving their written informed consent.

CTHRC1 expression and Kaplan-Meier survival curves analysis in data from The Cancer Genome Atlas (TCGA) database. Chandrashekar et al (28) reported an analysis platform on (http://ualcan.path.uab.edu/analysis.html) using the TCGA database. 546 EC and 35 normal tissue specimens were analyzed on the platform, where the mRNA expression levels of CTHRC1 were then compared between normal and EC groups from the TCGA database on the website, as previously described (28). The significance threshold was set at $\mathrm{P}<0.05$ following t-test. Kaplan-Meier survival curve analysis of 162 high CTHRC1 expression and 216 low CTHRC1 expression EC patients was performed using OncoLnc (http://www. oncolnc.org) (29). To separate patients into high/low expression groups, the $30 \%$ upper percentile and $40 \%$ lower percentile, as calculated using ROC, were used as the cut-off. The significance threshold was set at $\mathrm{P}<0.05$ following a Mantel-Cox test.

Immunohistochemistry (IHC). A total of $18 \mathrm{EC}$ and 11 adjacent normal endometrial tissues, which were excised $2 \mathrm{~cm}$ from the tumor tissues, were obtained from female Chinese patients (Set 1; age range, 50-65 years; mean age, $59 \pm 2.77$ years) who had undergone surgical treatment between January 2017 and December 2019 at the Shanghai First Maternity and Infant Hospital (Shanghai, China). No patient had undergone endocrine therapy, radiotherapy or chemotherapy prior to surgery. Endometrial tissues were sliced into 5- $\mu \mathrm{m}$ thick sections and embedded in paraffin. Next, sections were mounted on poly-L-lysine-coated slides and then deparaffinized in xylene and dehydrated with gradient ethanol. Antigen retrieval was performed in $10 \mathrm{mM}$ citrate buffer ( $\mathrm{pH} \mathrm{6.0)}$ for $10 \mathrm{~min}$. Endogenous peroxidase activity was blocked with $3 \%$ hydrogen peroxide for $10 \mathrm{~min}$ at room temperature. Following blocking with $1 \%$ horse serum albumin (Beijing Solarbio Science \& Technology Co., Ltd.) for $20 \mathrm{~min}$ at room temperature, the sections were incubated with rabbit polyclonal antibody against CTHRC1 (cat. no. ab85739; 1:100; Abcam) overnight at $4^{\circ} \mathrm{C}$ and then incubated with rabbit polyclonal secondary antibody (cat. no. ab205718; 1:20,000; Abcam) at $37^{\circ} \mathrm{C}$ for $30 \mathrm{~min}$. The sections were stained with DAB for $5 \mathrm{~min}$ and re-stained with hematoxylin for $2 \mathrm{~min}$ both at room temperature. The percentage of positive tumor cells was graded as follows: 0 , none; $1,1-25 \% ; 2,26-50 \%$; $3,51-75 \%$; and $4,76-100 \%$. Immunostaining intensity was rated as follows: 0 , none; 1 , weak; 2 , moderate; and 3 , intense. Specimens were considered immunopositive when $\geq 1 \%$ of the tumor cells had clear evidence of immunostaining. An IHC score was calculated for each specimen using the ImageJ software (version 1.8.0; National Institutes of Health), in which the percent positive rating was multiplied by the intensity rating. Each component of the tumor was scored independently by four investigators who were blinded to the tissue origin and the results were summed.

ELISA. Blood samples were obtained from a different set of 39 patients with EC and 12 healthy females (Set 2; age range, 40 - 65 years; mean age, $54 \pm 6.46$ years) who underwent surgical treatment between January and December 2018 at the Shanghai First Maternity and Infant Hospital. None of the patients had undergone radiotherapy, endocrine therapy or chemotherapy before surgery. The healthy individuals were visiting the hospital for a health examination. The blood was obtained from the elbow vein and was centrifuged for $10 \mathrm{~min}$ at $851 \mathrm{xg}$ at $4^{\circ} \mathrm{C}$ without anticoagulant, following which the serum samples were collected. The serum was frozen for storage at $-80^{\circ} \mathrm{C}$. The expression level of CTHRC1 was measured using a CTHRC1 ELISA kit (cat. no. EK13502; Signalway Antibody LLC), following the manufacturer's instructions.

Cell culture and treatment. Human EC cell lines Ishikawa and ECC1 were acquired from the Cell Bank of Shanghai Institute of Cell Biology, Chinese Academy of Sciences. ECC1 cells were authenticated by STR profiling and the locations of the cell samples were matched with the STR data of ECC-1 cells found in the databases of the American Type Culture Collection, Japanese Collection of Research Bioresources Cell Bank and Riken BioResource Research Center. Ishikawa and ECC1 cells were cultured in Dulbecco's modified Eagle's Medium/Nutrient Mixture F12 (DMEM/F12; Biological Industries) and RPMI-1640 medium (1640; Biological Industries), respectively, each supplemented with $100 \mathrm{U} / \mathrm{ml}$ penicillin/streptomycin (Gibco; Thermo Fisher Scientific, Inc.) and $10 \%$ fetal bovine serum (FBS; Biological Industries). The cells were cultured at $37^{\circ} \mathrm{C}$ in a $5 \% \mathrm{CO}_{2}$ humidified incubator. The Parental Cells group was cultured as aforementioned without any treatment for use to show the basal expression level of CTHRC1 via western blotting. Ishikawa and ECC1 cells cultured in complete medium treated with PBS or recombinant protein CTHRC1 $(100 \mu \mathrm{g} / \mathrm{ml}$; Abcam), CTHRC1 with the FAK signaling inhibitor defactinib $(1 \mu \mathrm{M}$; cat. no. HY-12289; MedChemExpress) or recombinant protein CTHRC1 $(100 \mu \mathrm{g} / \mathrm{ml})$ with the FAK signaling inhibitor Y15 (1 $\mu \mathrm{M}$; cat. no. HY-12444; MedChemExpress) were termed Control, +CTHRC1, +CTHRC1 +Defactinib or +CTHRC1 $+\mathrm{Y} 15$. All drug treatments were performed at $37^{\circ} \mathrm{C}$. Both cell lines were tested negative for mycoplasma.

Cell transfection. To overexpress human CTHRC1 in the Ishikawa and ECC1 EC cell lines, the coding sequence (5'-ATGCGACCCCAGGGCCCCGC-3') of CTHRC1 was cloned into a lentiviral vector with Ubi-MCS-3FLAG-SV40-puromycin using the Champion ${ }^{\mathrm{TM}}$ pET160 Gateway ${ }^{\mathrm{TM}}$ Expression Kit with Lumio ${ }^{\mathrm{TM}}$ Technology according to manufacturer's protocols (Invitrogen; Thermo Fisher Scientific, Inc.). The Gateway cloning protocols were followed. Both cell lines were infected with non-target or CTHRC1-specific lentiviral particles in 12-well plates with polybrene $(6 \mathrm{mg} / \mathrm{ml}$; Beijing Solarbio Science \& Technology Co., Ltd.). Puromycin $(1 \mu \mathrm{g} / \mathrm{ml})$ was added to the cells 
to generate stable CTHRC1-overexpressing clones. The experimental groups were termed negative control (NC) and CTHRC1 overexpressing (CTHRC1-OE).

Protein extraction and western blotting. To explore protein expression, radioimmunoprecipitation assay buffer (Beyotime Institute of Biotechnology) with a $1 \%$ dilution of the protease inhibitor phenylmethanesulfonyl fluoride (Beyotime Institute of Biotechnology) were used to lyse the parental cells, NC, CTHRC1-OE, control, +CTHRC1, +CTHRC1+Defactinib and $+\mathrm{CTHRC} 1+\mathrm{Y} 15$ cells. The concentration of extracted protein was determined using a BCA protein assay kit (Thermo Fisher Scientific, Inc.). The protein samples were mixed with loading buffer and then boiled for $10 \mathrm{~min}$. A total of $20 \mu \mathrm{g}$ protein were loaded into each lane of an 10\% SDS-PAGE gel for protein separation and then transferred to polyvinylidene fluoride membranes. The membranes were blocked with $5 \%$ bovine serum albumin (Sangon Biotech Co., Ltd.) for $1 \mathrm{~h}$ at room temperature and incubated with antibodies against CTHRC1 (cat. no. ab85739; 1:1,000; Abcam), Phosphorylated (p)-FAK ${ }^{\mathrm{Ty} 397}$ (p-FAK ${ }^{\text {Tyr397}}$; cat. no. 8556; 1:1,000; Cell Signaling Technology, Inc.) or Total-FAK (T-FAK; cat. no. 3285; 1:1,000; Cell Signaling Technology, Inc.) overnight at $4^{\circ} \mathrm{C}$. The membranes were incubated with peroxidase-linked secondary anti-rabbit antibody (cat. no. 7074; 1:2,000; Cell Signaling Technology, Inc.) for $1 \mathrm{~h}$ at room temperature to detect the bound primary antibodies, and the blotted proteins were visualized using Immobilon ${ }^{\text {TM }}$ Western Chemiluminescent HRP Substrate (EMD Millipore). The intensity of the protein bands was quantified using ImageJ software (version 1.8.0; National Institutes of Health). The relative expression of target proteins was described as a ratio relative to the expression of GAPDH and statistical data were based on at least three experimental replicates.

RNA extraction and reverse transcription-quantitative $P C R$ (RT-qPCR). Fresh EC and adjacent non-tumor tissues (14 of each) were acquired from female patients (Set 3; age range, 40-65 years; mean age, $53 \pm 6.21$ years) who underwent surgical treatment between January 2016 and December 2018 at the Shanghai First Maternity and Infant Hospital. The patients of Set 3 were different from Set 1 and Set 2. None of the patients had undergone radiotherapy, endocrine therapy, or chemotherapy before surgery. TRIzol ${ }^{\circledR}$ reagent (Thermo Fisher Scientific, Inc.) was used to extract the total RNA. RT-qPCR was performed as previously described (30). One Step TB Green ${ }^{\circledR}$ PrimeScript ${ }^{\mathrm{TM}}$ RT-qPCR kit (Takara Bio, Inc.) was used for RT-qPCR assays with the CFX96 Real Time-PCR Detection System (Bio-Rad Laboratories, Inc.). RT-qPCR was performed under the following thermocycling conditions: $30 \mathrm{sec}$ at $95^{\circ} \mathrm{C}$ followed by 40 cycles of $5 \mathrm{sec}$ at $95^{\circ} \mathrm{C}, 20 \mathrm{sec}$ at $60^{\circ} \mathrm{C}$ and $10 \mathrm{sec}$ at $72^{\circ} \mathrm{C} ; 1 \mathrm{~min}$ at $95^{\circ} \mathrm{C}, 30 \mathrm{sec}$ at $60^{\circ} \mathrm{C}$; and $30 \mathrm{sec}$ at $95^{\circ} \mathrm{C}$. Primer sequences were as follows: CTHRC1 forward, 5'-AGGGCTGCTTTTAACTCTGGT-3' and reverse, 5'-AGGGCTGCTTTTAACTCTGGT-3'; $\beta$-actin forward, 5'-AGCCTCGCCTTTGCCGAT-3' and reverse, 5'-CTTCTGACCCATGCCCACC-3'. All experiments were performed at least three times. The relative mRNA expression level was measured using the $2^{-\Delta \Delta C q}$ method (31).

Cell proliferation assay. Cell proliferation was assessed by Cell Counting Kit-8 (CCK-8) assay (Dojindo Molecular
Technologies, Inc.). Ishikawa and ECC1 cells were transfected first as aforementioned and then seeded into 96-well plates (4,000 cells/well). For CTHRC1 treatment, Ishikawa and ECC1 cells were seeded into a 96-well plate (3,000 cells/well) and treated with either PBS (Control) or the recombinant protein CTHRC1 $(100 \mu \mathrm{g} / \mathrm{ml}$; +CTHRC1) in complete medium. After 0,24 and $48 \mathrm{~h}, 10 \mu \mathrm{l}$ of CCK-8 solution was added to each well, followed by further incubation of the plates at $37^{\circ} \mathrm{C}$ for 4 h. Subsequently, a microplate reader (Molecular Devices, LLC) was used to measure the ultraviolet absorbance of all samples at $450 \mathrm{~nm}$. All experiments were performed at least three times.

Cell migration and invasion assays. For the migration assay, Ishikawa and ECC1 cells were transfected first as aforementioned, then harvested and re-suspended in serum-free DMEM/F12 and RPMI-1640 medium $\left(4 \times 10^{5}\right.$ cells $\left./ \mathrm{ml}\right)$, respectively, and then were placed into the upper Transwell ${ }^{\circledR}$ chamber $\left(8 \times 10^{4}\right.$ cells/well; pore size, $8 \mu \mathrm{m}$; Corning, Inc.). For CTHRC1 treatment, Ishikawa and ECC1 cells were harvested, re-suspended and then placed into the upper Transwell ${ }^{\circledR}$ chambers. PBS (Control) or recombinant protein CTHRC1 (100 $\mu \mathrm{g} / \mathrm{ml}$; +CTHRC1) or recombinant protein CTHRC1 (100 $\mu \mathrm{g} / \mathrm{ml})$ with defactinib $(1 \mu \mathrm{M} ;+$ CTHRC1+Defactinib) or recombinant protein CTHRC1 $(100 \mu \mathrm{g} / \mathrm{ml})$ with Y15 (1 $\mu \mathrm{M} ;+$ CTHRC1+Y15) were added to both the upper and bottom chambers. For invasion assay, $50 \mu 1$ Matrigel matrix (1:5; pre-diluted with serum-free medium; incubated at $4^{\circ} \mathrm{C}$ for pre-coating liquid and at $37^{\circ} \mathrm{C}$ for $30 \mathrm{~min}$ for gelation; BD Biosciences) was put onto the upper chamber and $2 \times 10^{5}$ transfected Ishikawa and ECC1 cells were seeded into the Matrigel matrix, which was pre-coated at $37^{\circ} \mathrm{C}, 2 \mathrm{~h}$ later. For CTHRC1 treatment, Ishikawa and ECC1 cells were seeded into the Matrigel matrix as aforementioned and treated with either Control or +CTHRC1. After that, $800 \mu \mathrm{l}$ DMEM/F12 and RPMI-1640 medium supplemented with $20 \%$ FBS were added into the lower chamber. After $16 \mathrm{~h}$ (migration assay) or $24 \mathrm{~h}$ incubation (invasion assay with Matrigel pre-coating) at $37^{\circ} \mathrm{C}$, cells were stained with Calcein-AM $(0.2 \mu \mathrm{g} / \mathrm{ml}$; cat. no. C3100MP; Invitrogen; Thermo Fisher Scientific, Inc.) for $30 \mathrm{~min}$ at $37^{\circ} \mathrm{C}$. Leica DMi8 inverted fluorescent microscope (magnification, x200; Leica Microsystems $\mathrm{GmbH}$ ) was used to observe cell migration and invasion. The number of cells that had migrated or invaded was counted using MetaMorph ${ }^{\circledR}$ Microscopy Automation and Image Analysis Software (version 2.5; Molecular Devices, LLC). Five fields of view were imaged per chamber. Each migration and invasion assay was repeated three times on different days with different batches of cells. All experiments were performed at least three times.

Wound healing assay. Ishikawa and ECC1 cells were transfected first as aforementioned and seeded into 6-well plates at a concentration of $1 \times 10^{6}$ cells/well until they reached $\geq 90 \%$ confluence. The monolayer was scratched with a sterile $200 \mu \mathrm{l}$ pipette tip and then cell debris was removed using PBS three times, followed by incubation in serum-free medium. For CTHRC1 treatment, Ishikawa and ECC1 cells were seeded as aforementioned and treated with PBS (Control) or recombinant protein CTHRC1 $(100 \mu \mathrm{g} / \mathrm{ml}$; + CTHRC1) or recombinant protein CTHRC1 $(100 \mu \mathrm{g} / \mathrm{ml})$ 

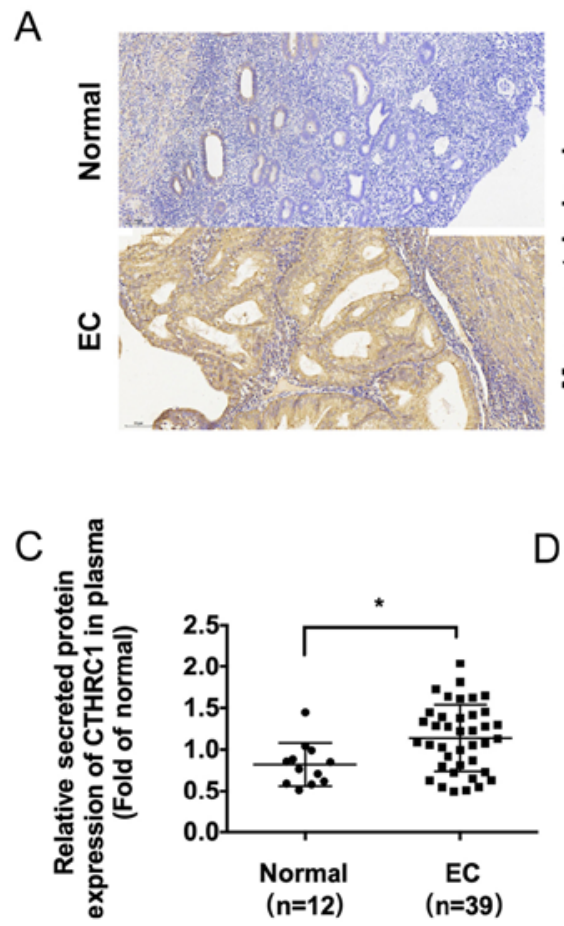

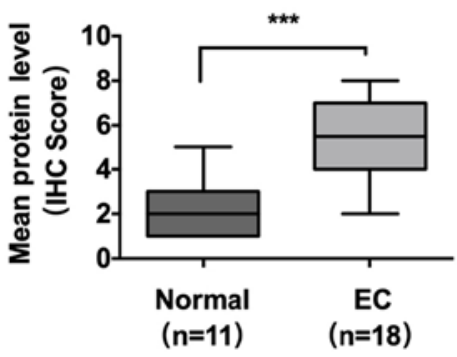

D

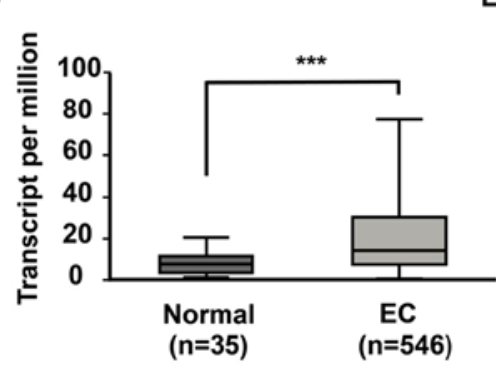

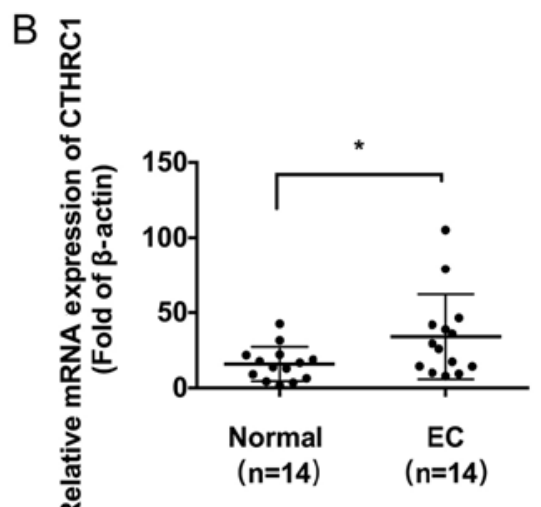

$\mathrm{E}$

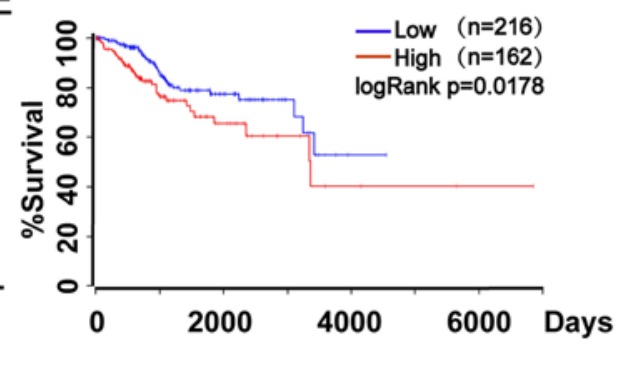

Figure 1. CTHRC1 is commonly upregulated in EC. (A) Immunohistochemical analysis of CTHRC1 expression in adjacent normal endometrium (n=11) and EC ( $\mathrm{n}=18)$ tissues. Magnification, x200. No or weak expression of CTHRC1 was observed in normal endometrium tissues. Strong cell membrane and cytoplasmic expression of CTHRC1 was observed in most EC tissues. (B) Relative RNA expression levels of CTHRC1 in EC tissues (n=14) and adjacent normal tissues $(n=14)$ were assessed by reverse transcription-quantitative PCR analysis. (C) ELISA analysis of levels of secreted CTHRC1 in the plasma of EC patients or normal females (EC group, $n=39$; normal group, $n=12$ ). (D) Relative mRNA expression of CTHRC1 in the normal and EC groups in TCGA Uterine Corpus Endometrial Cancer database (EC tissues, $n=546$; normal tissues, $n=35$ ). (E) Kaplan-Meier curves of survival for EC patients with high or low CTHRC1 expression (high CTHRC1 expression, $\mathrm{n}=162$; low CTHRC1 expression, $\mathrm{n}=216$ ). Experiments were repeated in triplicate. Data are presented as the mean \pm SD. ${ }^{*} \mathrm{P}<0.05,{ }^{* * *} \mathrm{P}<0.001$. CTHRC1, collagen triple helix repeat containing 1; EC, endometrial cancer; TCGA, The Cancer Genome Atlas.

with defactinib $(1 \mu \mathrm{M}$; +CTHRC1+Defactinib) or recombinant protein CTHRC1 $(100 \mu \mathrm{g} / \mathrm{ml})$ with $\mathrm{Y} 15(1 \mu \mathrm{M}$; $+\mathrm{CTHRC} 1+\mathrm{Y} 15)$ in complete medium until they reached $\geq 90 \%$ confluence. The cells were incubated in serum-free medium treated with Control or +CTHRC1 or +CTHRC1+Defactinib or +CTHRC1+Y15 after scratching. The margin of the wound was detected using a Leica DMi8 inverted light microscope (magnification, x50; Leica Microsystems $\mathrm{GmbH}$ ) at 0 and $24 \mathrm{~h}$. In total, four fields of view were imaged for every well. All experiments were repeated at least three times.

Cell immunofluorescence. Ishikawa and ECC1 cells were transfected first as aforementioned, following which they were seeded into $35 \mathrm{~mm}$ confocal dishes and cultured in complete medium overnight. For CTHRC1 treatment, Ishikawa and ECC1 cells were seeded as above and cultured in complete medium treated with PBS (Control) or recombinant protein CTHRC1 $(100 \mu \mathrm{g} / \mathrm{ml}$; +CTHRC1) overnight. Cells were then fixed with $4 \%$ paraformaldehyde for $10 \mathrm{~min}$ at room temperature, permeabilized with $0.1 \%$ Triton $\mathrm{X}-100$ for $10 \mathrm{~min}$ at room temperature and blocked with 5\% goat serum (Beijing Solarbio Science \& Technology Co., Ltd.) for $30 \mathrm{~min}$ at room temperature before incubation with antibody against vinculin (cat. no. ab129002; 1:100; Abcam) at $4^{\circ} \mathrm{C}$ overnight. The cells were then incubated with Alexa Fluor ${ }^{\circledR} 555$ secondary antibody (cat. no. P0179; 1:1,000; Beyotime Institute of Biotechnology) for $1 \mathrm{~h}$ at room temperature and then phalloidin-FITC (cat. no. C1033; 1:100; Beyotime Institute of Biotechnology) for
$30 \mathrm{~min}$ at room temperature. Cells were counterstained with DAPI (cat. no. C1002; 1:500; Beyotime Institute of Biotechnology) for $2 \mathrm{~min}$ at room temperature before being analyzed using a confocal microscope (magnification, x100; Leica TCS SP8; Leica Microsystems GmbH).

Statistical analysis. SPSS version 23.0 (IBM Corp.) was used for statistical analysis and graphs were edited using GraphPad Prism version 6.0 (GraphPad Software, Inc.). Measurement data were analyzed using unpaired or paired Student's t-test, or one-way analysis of variance, as appropriate. Multiple comparisons with equal variances assumed were performed by Tukey's post hoc test. Each experiment was performed in triplicate. All data are presented as the mean \pm SD. Differences were considered statistically significant at $\mathrm{P}<0.05$.

\section{Results}

CTHRC1 is expressed in EC tissues. To verify CTHRC1 expression patterns, IHC analysis was performed on samples from EC $(n=18)$ and normal endometrium $(n=11)$ tissues. IHC results showed stronger CTHRC1 staining in the representative EC tissues than in the normal endometrial tissues $(\mathrm{P}<0.001$; Fig. 1A). To explore the expression of CTHRC1 in $\mathrm{EC}$, the relative RNA expression levels of CTHRC1 were compared in adjacent normal tissues $(n=14)$ and EC tissues $(n=14)$ using RT-qPCR. CTHRC1 expression was significantly increased in EC tissues compared with normal tissues $(\mathrm{P}<0.05$; 
Fig. 1B). ELISA was performed to determine the concentration of secreted CTHRC1 in plasma. CTHRC1 release was significantly increased in the EC group $(n=39)$ compared to the normal group $(n=12)(\mathrm{P}<0.05$; Fig. $1 \mathrm{C})$. The results indicated that the expression levels of CTHRC1 in EC tissues were also significantly increased compared to normal tissues $(\mathrm{P}<0.001$; Fig. 1D). Furthermore, patients where EC samples had high CTHRC1 expression had worse survival rates than patients where EC samples had low CTHRC1 expression $(\mathrm{P}<0.05$; log-rank; Fig. 1E).Taken together, these results indicated that CTHRC1 was significantly increased in EC.

Overexpression of CTHRC1 enhances the migration of EC cells in vitro. To explore the effect of CTHRC1 on EC, CTHRC1 was overexpressed in Ishikawa and ECC1 cells. CTHRC1 expression was analyzed by western blotting (Fig. 2A). CCK-8 assay was performed to investigate the proliferative ability of Ishikawa and ECC1 cells. There was no significant difference in proliferation between the NC cells and CTHRC1-OE cells in both Ishikawa and ECC1 cell lines (Fig. 2B). To explore the role of CTHRC1 on the migration of Ishikawa and ECC1 cells, Transwell migration assays were performed. As shown in Fig. 2C, compared with cells transfected with the empty vector, migration was significantly increased in the CTHRC1-OE cells. In addition, the relationship between CTHRC1 and cell migration was investigated by wound healing assays. The results demonstrated that cells in the CTHRC1-OE group showed more rapid wound closure ability compared with the NC group (Fig. 2D). Transwell invasion assays revealed that invasion was significantly increased in the CTHRC1-OE cells compared to the NC cells (Fig. 2E). Cells were stained for vinculin, a focal adhesion protein, and with phalloidin, a cytoskeletal protein dye, to display the focal adhesion and cytoactin remodeling. Confocal immunofluorescence staining revealed weaker vinculin and stronger phalloidin staining in CTHRC1-OE cells compared with the NC (Fig. 2F). These results indicated that overexpression of CTHRC1 could promote the migration of EC cells. However, there was no significant influence on cell proliferation in vitro.

Recombinant CTHRC1 promotes EC cell migration in vitro. Ishikawa and ECC1 cells were treated with recombinant CTHRC1 protein to investigate the effect of CTHRC1 on EC. CCK-8 assays were performed to investigate the proliferation ability of Ishikawa and ECC1 cells. There was no significant difference in proliferation after treatment with $\mathrm{CTHRC1}$ protein in either Ishikawa or ECC1 cells (Fig. 3A). To investigate the effect of CTHRC1 on Ishikawa and ECC1 migration, Transwell migration assays were performed. Following CTHRC1 treatment, migration was significantly enhanced in both ECC1 and Ishikawa cells (Fig. 3B). Furthermore, wound healing assays were performed to investigate the migration of EC cells. Cells in the +CTHRC1 group showed more rapid wound closure ability compared with that in the Control group (Fig. 3C). Transwell invasion assays showed that invasion was significantly increased in +CTHRC1 compared with Control (Fig. 3D). Confocal immunofluorescence staining revealed weaker vinculin and stronger phalloidin staining in +CTHRC1 than that in Control (Fig. 3E). These results indicated that CTHRC1 treatment could enhance the migration of EC cells in vitro.
CTHRC1 mediates the migration of EC cells via the FAK signaling pathway. To explore the molecular mechanisms underlying CTHRC1 activity, the signaling pathways responsible for CTHRC1-mediated cell migration were investigated. P-FAK and T-FAK are both important factors in the FAK signaling pathway. The protein levels of P-FAK and T-FAK were examined by western blot analysis. Overexpression of CTHRC1 led to significantly increased expression of P-FAK compared to the NC cells (Fig. 4A). In addition, treatment with recombinant protein CTHRC1 also led to increased expression of P-FAK, however treatments on the +CTHRC1 cells with two FAK signaling inhibitors, defactinib and Y15, respectively, led to decreased expression of P-FAK compared to the +CTHRC1 group (Fig. 4B). Transwell migration assays and wound healing assays revealed that the two inhibitors of the FAK signaling pathway led to reduced cell migration compared with the +CTHRC1 group (Fig. 4C and D). These results suggested that $\mathrm{CTHRC1}$ mediated the migration of EC cells via the FAK signaling pathway (Fig. 4E).

\section{Discussion}

$\mathrm{EC}$ is one of the most common cancers in women. In addition, the incidence and mortality rates of EC are expected to rise both in developed and developing countries with the increasing aging population and prevalence of obesity (32). Treatment for metastatic EC is ineffective and metastasis is the major reason for relapse and death, since there is no effective therapy for this condition (33). Therefore, identifying the molecular mechanisms of the peritoneal metastatic process is crucial for developing novel preventive strategies. CTHRC1 has been reported to be involved in bone formation, vascular remodeling and morphogenesis (18-20). Previous studies indicated that CTHRC1 was overexpressed in human pancreatic cancer tissues, hepatocellular carcinoma, colorectal cancer and gastrointestinal stromal tumors (34-37). Overexpression of CTHRC1 is reported during tumor invasion and metastasis (27). where it can promote migration by activating FAK signaling in pancreatic cancer (16). However, the role of CTHRC1 in EC is still unknown. The results of the present study suggest that CTHRC1 is highly expressed in EC and can promote the migration of EC cells in vitro. The present study also indicated that CTHRC1 mediates the migration of EC cells via the FAK signaling pathway.

Expression levels of CTHRC1 were compared between EC tissues and normal tissues and it was observed that CTHRC1 levels were significantly higher in EC tissues than in normal and precancerous tissues. Meanwhile, secreted CTHRC1 in plasma was significantly increased in EC patients compared with controls. Data from the TCGA database were consistent with these findings and indicated that high CTHRC1 expression predicted a poor prognosis in EC. To investigate the role of CTHRC1 in EC, CTHRC1 was overexpressed in Ishikawa and ECC1 cells, two standard cellular models of EC. The effects of CTHRC1 were examined via Transwell migration assays, wound healing assays and Transwell invasion assays with Matrigel. The results indicated that CTHRC1 could promote cell migration in vitro. However, CCK-8 assays showed that there was no significant effect of CTHRC1 on cell proliferation. It has been shown that as carcinomas 
A

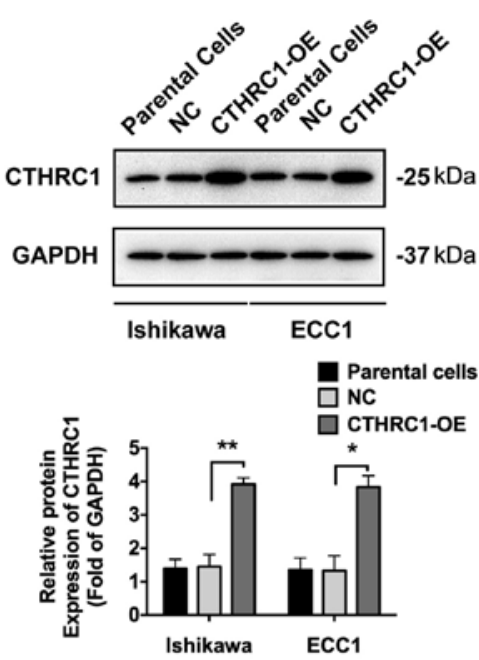

D

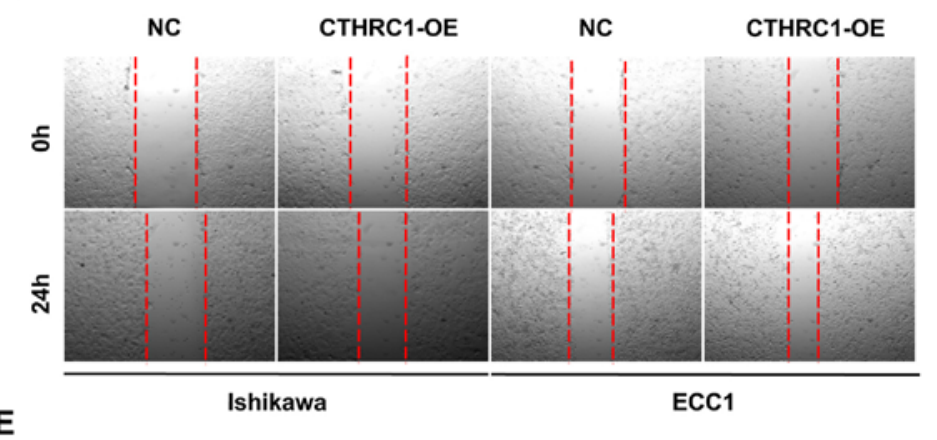

C C NC $\quad$ CTHRC1-OE

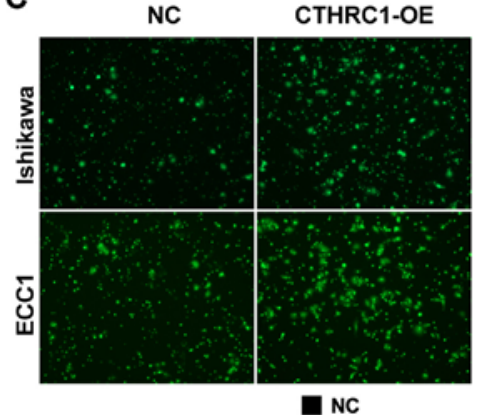

$\rightarrow$ NC
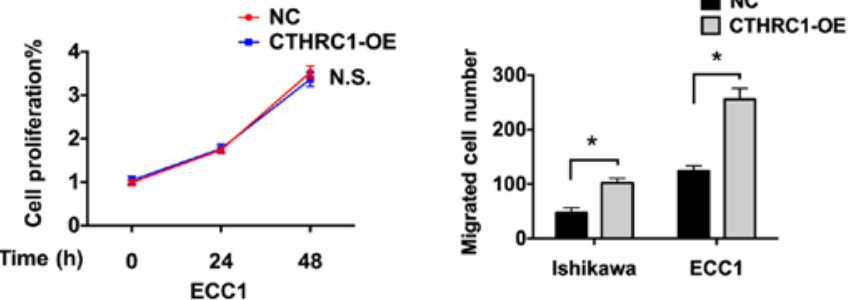

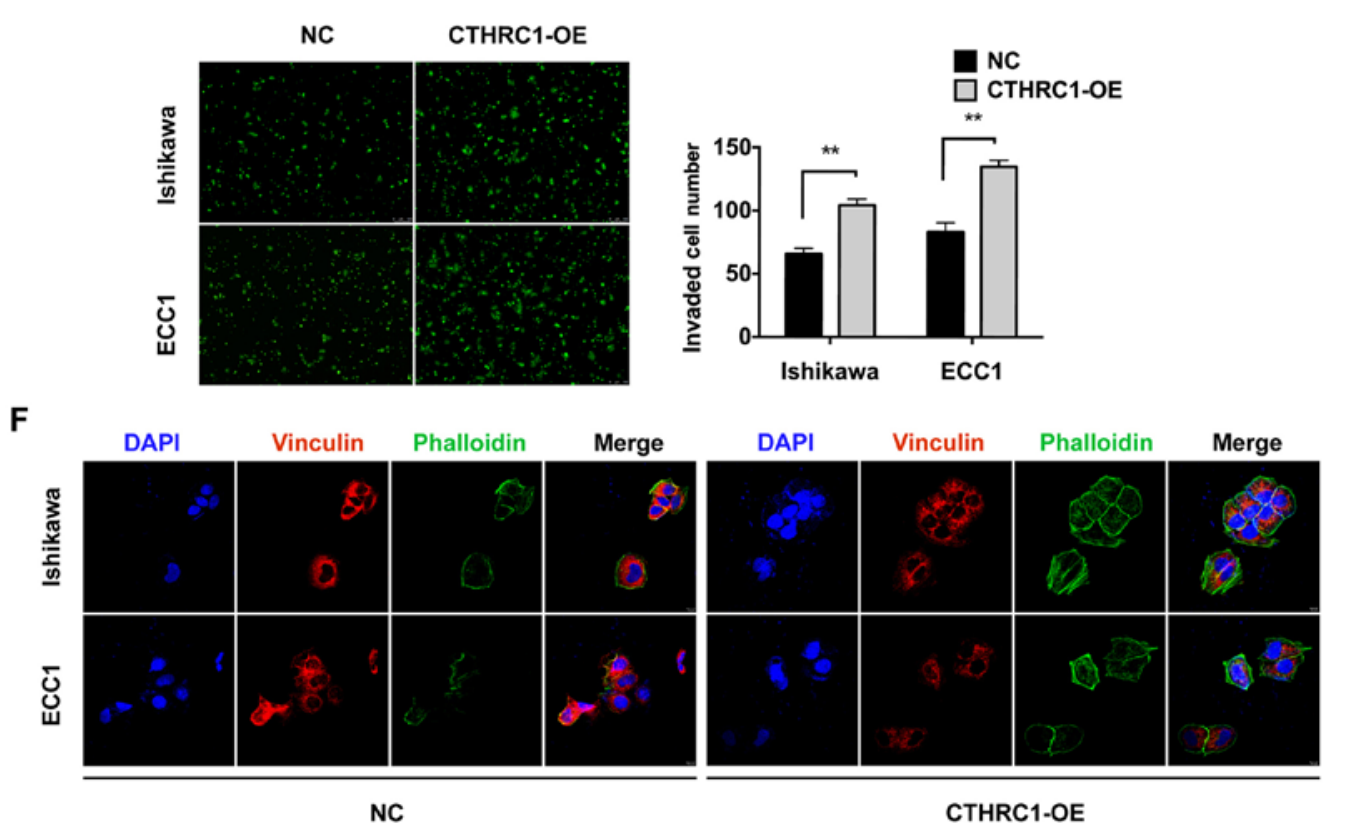

Figure 2. Overexpression of CTHRC1 promotes the migration of EC cells in vitro. (A) Western blot analysis of the expression levels of CTHRC1 in Ishikawa and ECC1 cells. (B) Proliferation of Ishikawa and ECC1 cells with CTHRC1-OE was confirmed by CCK-8 assays. (C) Migration of Ishikawa and ECC1 cells with CTHRC1-OE confirmed by Transwell assays. Magnification, x200. (D) Migration of Ishikawa and ECC1 cells with CTHRC1-OE confirmed by wound healing assays. Magnification, x50. (E) Invasion of Ishikawa and ECC1 cells with CTHRC1-OE confirmed by Transwell assays with Matrigel. Magnification, x200). (F) Focal adhesion and actin remodeling were analyzed by immunofluorescence. Magnification, x100. Vinculin was marked by red fluorescence and phalloidin, an actin stain, was marked by green fluorescence, while the nucleus was stained with DAPI (blue). The results were determined in triplicate. Data are presented as the mean $\pm \mathrm{SD} .{ }^{*} \mathrm{P}<0.05,{ }^{* *} \mathrm{P}<0.01$. CTHRC1, Collagen triple helix repeat containing 1; EC, endometrial cancer; CCK-8, Cell Counting Kit-8; $\mathrm{OE}$, overexpression.

arising from epithelial tissues progress to higher pathological grades of malignancy, reflected in local invasion and distant metastasis, the associated cancer cells typically develop alterations in their shape as well as in their attachment to 
A

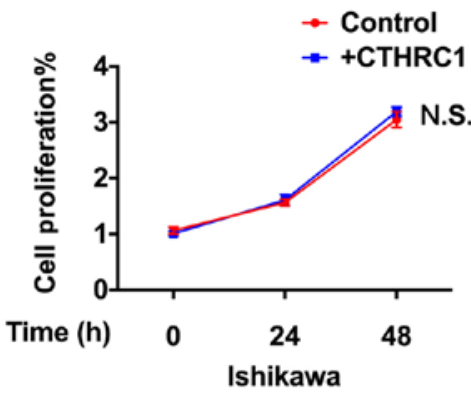

B

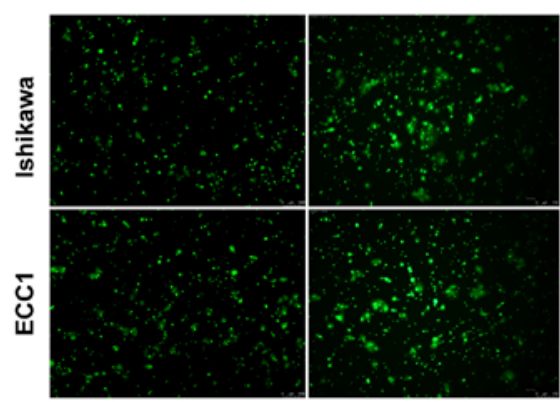

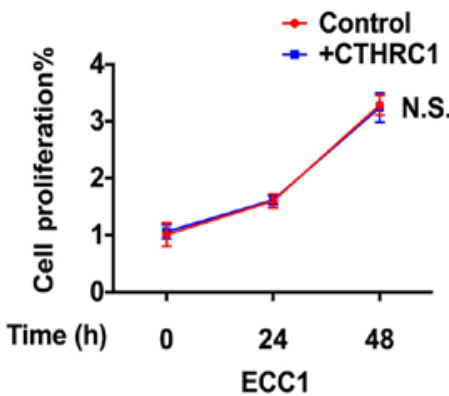

Control

+CTHRC1

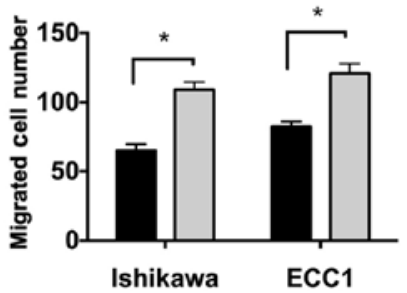

C

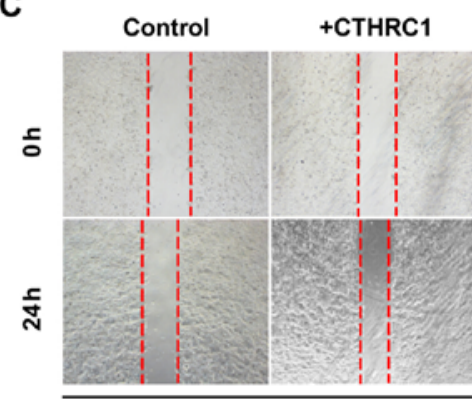

D

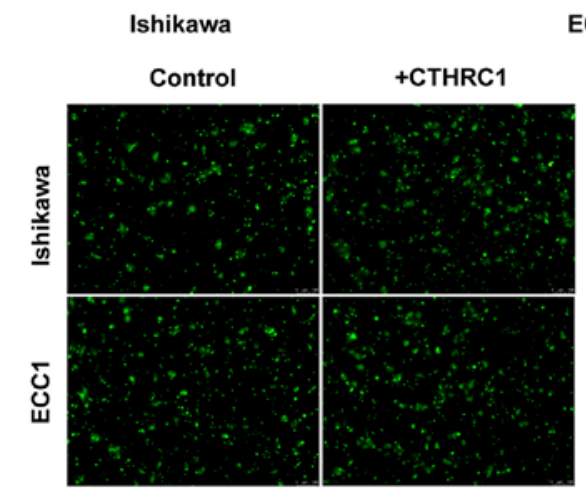

E

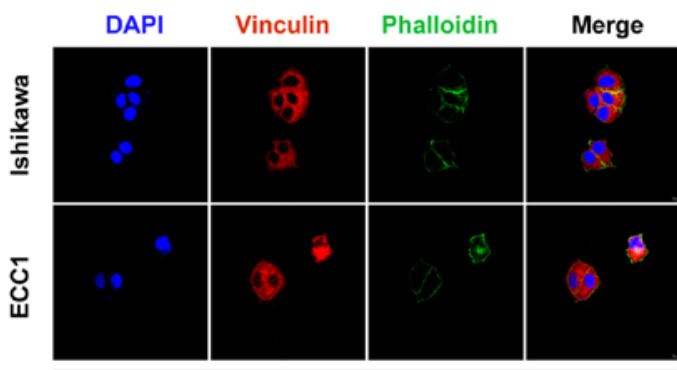

Control

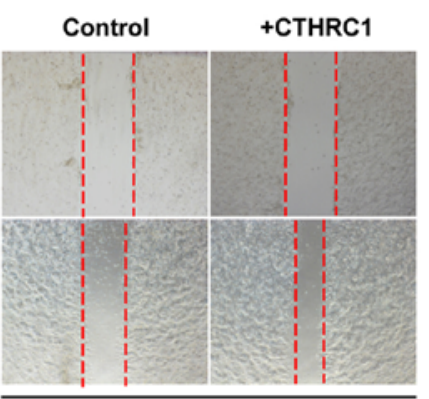

ECC1

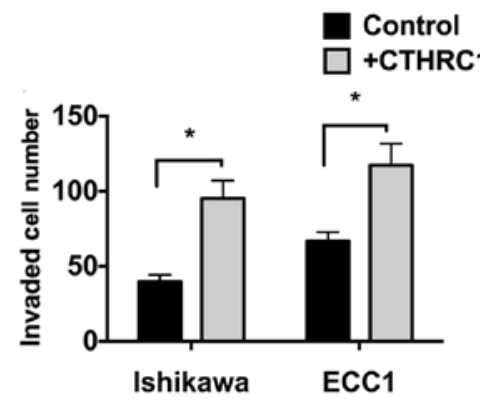

Control

$\square+$ CTHRC1

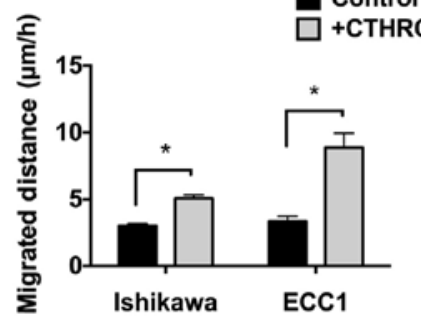

Ishikawa

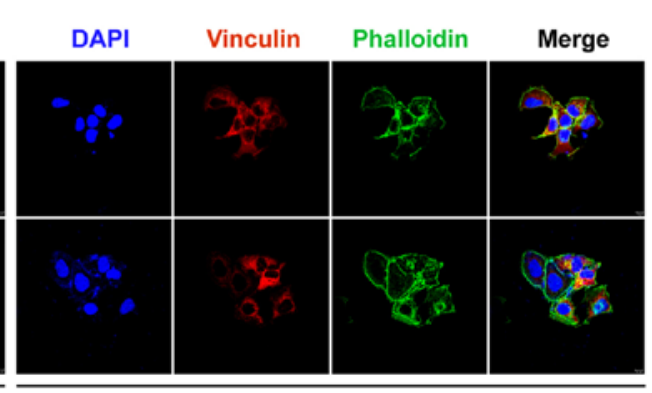

+CTHRC1

Figure 3. Recombinant CTHRC1-mediated promotion of EC cell migration in vitro. (A) Proliferation of +CTHRC1 Ishikawa and ECC1 cells confirmed by CCK-8 assays. (B) Migration of Ishikawa and ECC1 cells with +CTHRC1 confirmed by Transwell assays. Magnification, x200. (C) Migration of Ishikawa and ECC1 cells with +CTHRC1 confirmed by wound healing assays. Magnification, x50. (D) Invasion of Ishikawa and ECC1 cells with +CTHRC1 confirmed by Transwell assays with Matrigel. Magnification, x200. (E) Focal adhesion and cyto-actin remodeling were analyzed by immunofluorescence. Magnification, x100. Vinculin was marked by red fluorescence and phalloidin was marked by green fluorescence, while the nucleus was stained with DAPI (blue). The experiments were performed in triplicate, data are presented as the mean $\pm \mathrm{SD}$. ${ }^{*} \mathrm{P}<0.05$. CTHRC1, Collagen triple helix repeat containing 1 ; EC, endometrial cancer; CCK-8, cell counting kit-8; +CTHRC1, cells with recombinant CTHRC1 protein treatment. 
A
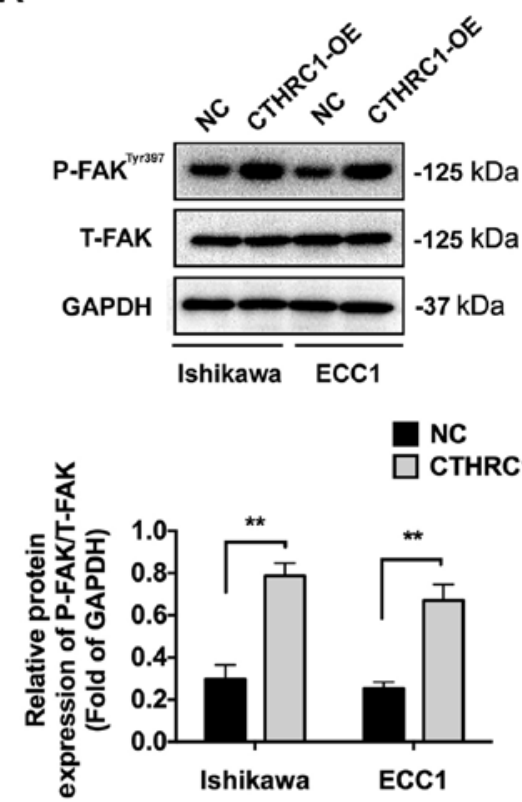

C

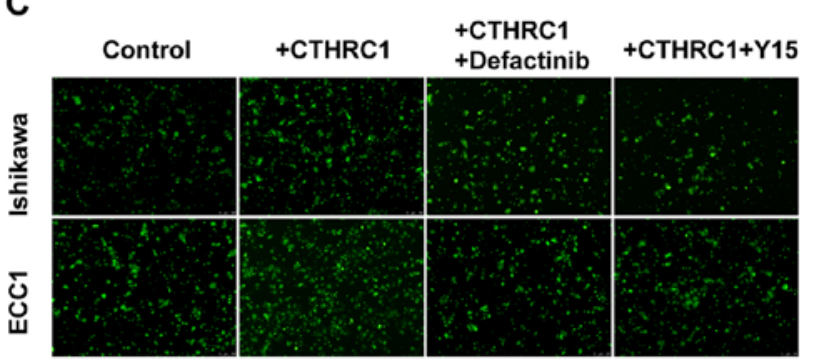

D

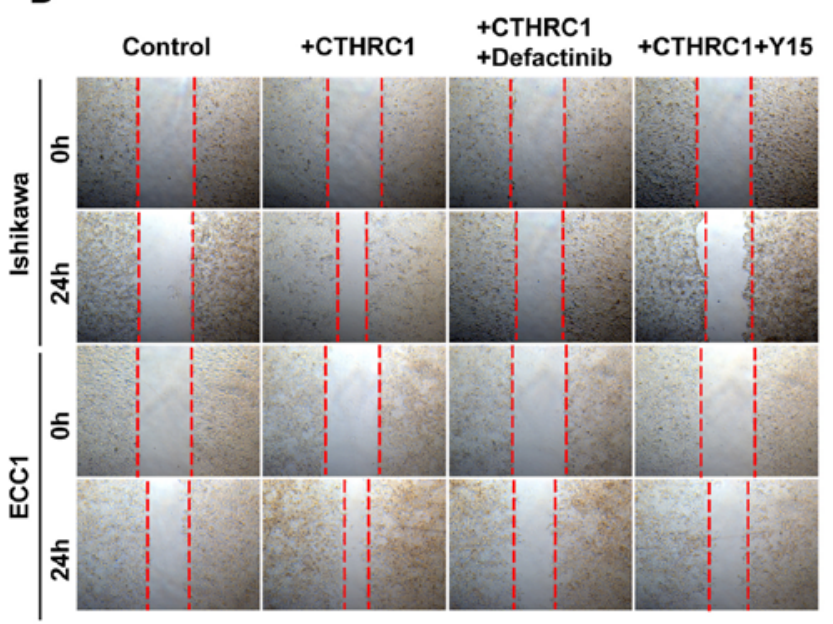

B
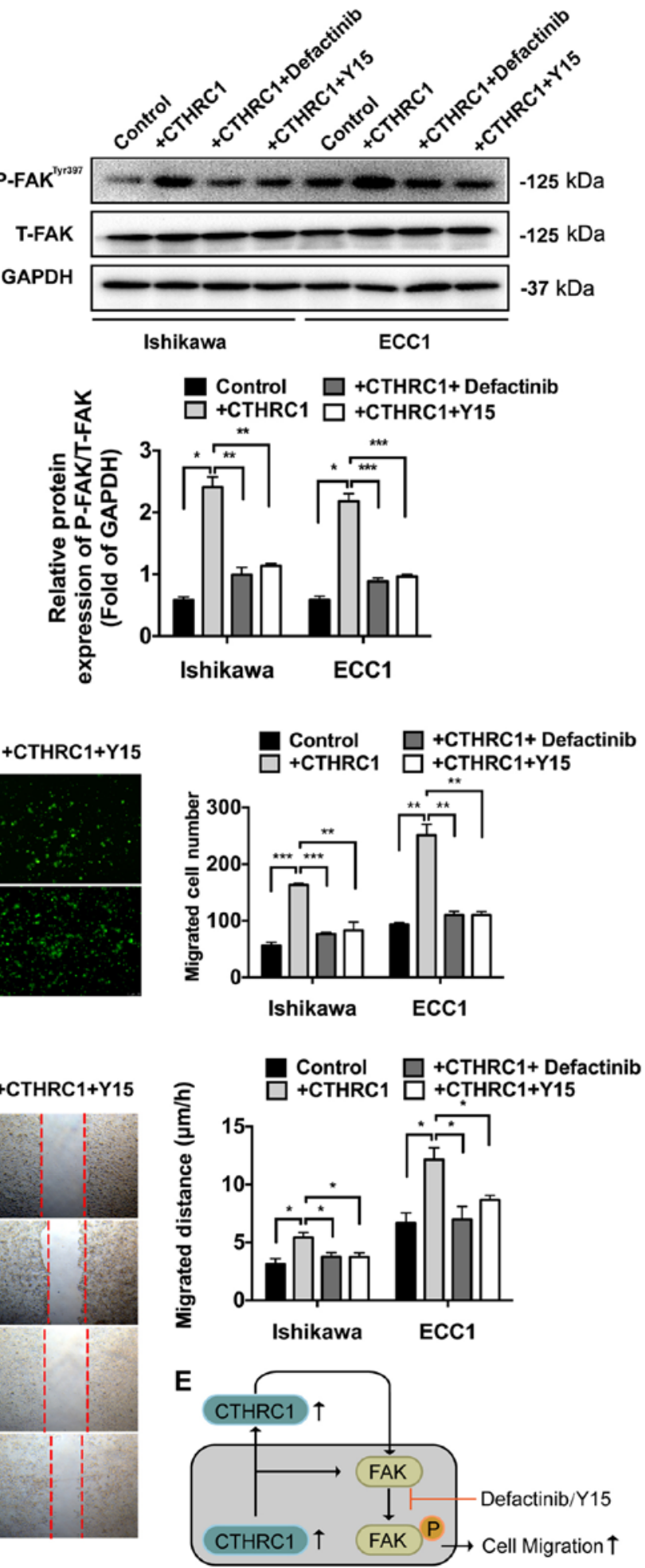

Figure 4. CTHRC1 mediates the migration of EC cells via the FAK signaling pathway. (A) Western blot analysis of P-FAK ${ }^{\text {Tyr397 }}$ and T-FAK in cells with CTHRC1-OE. (B) Western blot analysis of P-FAK ${ }^{\text {Tyr397 }}$ and T-FAK in the +CTHRC1 group or +CTHRC1 cells with defactinib or Y15. Migration confirmed by (C) Transwell assays, magnification, x200; and (D) wound healing assays. Magnification, x50. (E) The high expression of CTHRC1 in EC cells and plasma promoted EC cell migration by activating the FAK signaling pathway. The FAK signaling inhibitors, defactinib and Y15, could reverse the enhanced migration mediated by CTHRC1. The experiments were performed in triplicate. Data are presented as the mean $\pm \mathrm{SD}$. ${ }^{*} \mathrm{P}<0.05$, ${ }^{* *} \mathrm{P}<0.01,{ }^{* * *} \mathrm{P}<0.001$. CTHRC1, collagen triple helix repeat containing 1; EC, endometrial cancer; FAK, focal adhesion kinase; P-FAK, phospho-FAK; T-FAK, Total-FAK; OE, overexpression.

other cells and to the ECM (38). Expression of genes encoding cell-cell and cell-ECM adhesion molecules is demonstrably altered in certain highly aggressive carcinomas, with those favoring cytostasis typically being downregulated (39). To investigate the focal adhesion and cytoactin remodeling of the
CTHRC1-OE group, immunofluorescence was performed to stain for vinculin, a focal adhesion protein, and for filamentous actin, using cytoskeletal protein dye phalloidin. The results indicated downregulated focal adhesion protein and an altered cytoskeleton. Furthermore, the effect of CTHRC1 
was demonstrated via CTHRC1 protein treatment. CTHRC1 protein could promote migration in vitro, but had no effect on cell proliferation. Immunofluorescence showed analogous changes in the $+\mathrm{CTHRC1}$ group. Evidence to date supports the notion that CTHRC1 integrates multiple aggressive signaling pathways $(40,41)$. The FAK signaling pathway is known for its essential role in integrin signaling and overexpressed in many human tumors. It is also important in a variety of cellular functions such as growth, survival, invasion and migration (13-15). Furthermore, in previous studies, it was shown that migration of cancer cells was decreased after inhibiting the activation of FAK signaling $(16,17)$. Overexpression of FAK has a potential role in the initiation of metastatic signaling events (12). To further demonstrate the underlying molecular mechanism of CTHRC1 in EC migration, the FAK signaling pathway was investigated. Overexpression or treatment with CTHRC1 could promote an increase in the levels of P-FAK. Furthermore, the two FAK signaling inhibitors, defactinib and Y15, reversed the enhanced migration and the increased P-FAK levels mediated by CTHRC1, respectively, indicating that CTHRC1 mediated the migration of EC cells via the FAK signaling pathway. Therefore, the present findings are the first to suggest a role for CTHRC1 in affecting the migration of EC cells through the FAK signaling pathway.

In conclusion, the results of the present study indicated that CTHRC1 was expressed at a higher level in EC tissues than in normal tissues. Additionally, they suggested that CTHRC1 may promote the migration of EC cells in vitro via the FAK signaling pathway. Therefore, the CTHRC1/FAK signaling pathway may be a potential therapeutic target for the treatment of EC.

\section{Acknowledgements}

Not applicable.

\section{Funding}

This study was supported by the National Natural Science Foundation of China (grant no. 81672574) and the Outstanding Youth Projects of Shanghai Municipal Commission of Health and Family Planning (grant no. 2018YQ23).

\section{Availability of data and materials}

The datasets used and/or analyzed in the present study are available from the corresponding author on reasonable request. The datasets generated and/or analyzed during the current study are available in the TCGA repository and the results shown here are in whole or part based upon data generated by the following website: https://ualcan.path.uab.edu/analysis. html.

\section{Authors' contributions}

$\mathrm{XH}$ and $\mathrm{YB}$ performed all experiments and wrote the manuscript. XWe, MW and YL were responsible for data processing, figure processing and statistical analysis. XWa designed the study, approved the final version to be published and agreed to be accountable for all aspects of the work. All authors read and approved the final manuscript.

\section{Ethics approval and consent to participate}

This research was approved by the Human Investigation Ethics Committee of the Shanghai First Maternity and Infant Hospital. Samples were collected from patients after receiving their written informed consent.

\section{Patient consent for publication}

Not applicable.

\section{Competing interests}

The authors declare that they have no competing interests.

\section{References}

1. Liu Y, Patel L, Mills GB, Lu KH, Sood AK, Ding L, Kucherlapati R, Mardis ER, Levine DA, Shmulevich I, et al: Clinical significance of CTNNB1 mutation and Wnt pathway activation in endometrioid endometrial carcinoma. J Natl Cancer Inst 106: 106, 2014.

2. Amant F, Moerman P, Neven P, Timmerman D, Van Limbergen E and Vergote I: Endometrial cancer. Lancet 366: 491-505, 2005.

3. Baum M, Budzar AU, Cuzick J, Forbes J, Houghton JH, Klijn JG and Sahmoud T; ATAC Trialists' Group: Anastrozole alone or in combination with tamoxifen versus tamoxifen alone for adjuvant treatment of postmenopausal women with early breast cancer: First results of the ATAC randomised trial. Lancet 359: 2131-2139, 2002.

4. Sasaki M, Dharia A, Oh BR, Tanaka Y, Fujimoto S and Dahiya R: Progesterone receptor $\mathrm{B}$ gene inactivation and $\mathrm{CpG}$ hypermethylation in human uterine endometrial cancer. Cancer Res 61: 97-102, 2001.

5. Daikoku T, Hirota Y, Tranguch S, Joshi AR, DeMayo FJ, Lydon JP, Ellenson LH and Dey SK: Conditional loss of uterine Pten unfailingly and rapidly induces endometrial cancer in mice. Cancer Res 68: 5619-5627, 2008.

6. Colombo N, Preti E, Landoni F, Carinelli S, Colombo A, Marini C, Sessa C, ESMO Guidelines Working Group: Endometrial cancer: ESMO Clinical Practice Guidelines for diagnosis, treatment and follow-up. Ann Oncol 24 (Suppl 6): vi33-38, 2013.

7. Yoney A, Yildirim C, Isikli L and Unsal M: Prognostic factors and treatment outcomes in patients with operated endometrial cancer: Analysis of 674 patients at a single institution. J BUON 16: 64-73, 2011

8. Su JL, Yang PC, Shih JY, Yang CY, Wei LH, Hsieh CY, Chou CH, Jeng YM, Wang MY, Chang KJ, et al: The VEGF-C/Flt-4 axis promotes invasion and metastasis of cancer cells. Cancer Cell 9: 209-223, 2006.

9. Holst F, Werner HMJ, Mjøs S, Hoivik EA, Kusonmano K, Wik E, Berg A, Birkeland E, Gibson WJ, Halle MK, et al: PIK3CA amplification associates with aggressive phenotype but not markers of AKT-mTOR signaling in endometrial carcinoma. Clin Cancer Res 25: 334-345, 2019.

10. Kodama J, Hasengaowa, Kusumoto T, Seki N, Matsuo T, Ojima Y, Nakamura K, Hongo A and Hiramatsu Y: Prognostic significance of stromal versican expression in human endometrial cancer. Ann Oncol 18: 269-274, 2007.

11. Lin Q, Chen H, Zhang M, Xiong H and Jiang Q: Knocking down FAM83B inhibits endometrial cancer cell proliferation and metastasis by silencing the PI3K/AKT/mTOR pathway. Biomed Pharmacother 115: 108939, 2019.

12. Alowayed N, Salker MS, Zeng N, Singh Y and Lang F: LEFTY2 controls migration of human endometrial cancer cells via focal adhesion kinase activity (FAK) and miRNA-200a. Cell Physiol Biochem 39: 815-826, 2016.

13. Nishita M, Enomoto M, Yamagata $\mathrm{K}$ and Minami $\mathrm{Y}$ : Cell/tissue-tropic functions of Wnt5a signaling in normal and cancer cells. Trends Cell Biol 20: 346-354, 2010.

14. Wang J, Wen T, Li Z, Che X, Gong L, Yang X, Zhang J, Tang H, He L, Qu X, et al: MicroRNA-1224 inhibits tumor metastasis in intestinal-type gastric cancer by directly targeting FAK. Front Oncol 9: 222, 2019. 
15. Balsas P, Palomero J, Eguileor A, Rodríguez ML, Vegliante MC, Planas-Rigol E, Sureda-Gómez M, Cid MC, Campo E and Amador V: SOX11 promotes tumor protective microenvironment interactions through CXCR4 and FAK regulation in mantle cell lymphoma. Blood 130: 501-513, 2017.

16. Jiang H, Liu X, Knolhoff BL, Hegde S, Lee KB, Jiang H, Fields RC, Pachter JA, Lim KH and DeNardo DG: Development of resistance to FAK inhibition in pancreatic cancer is linked to stromal depletion. Gut 69: 122-132, 2020.

17. Yamaura T, Kasaoka T, Iijima N, Kimura M and Hatakeyama S: Evaluation of therapeutic effects of FAK inhibition in murine models of atherosclerosis. BMC Res Notes 12: 200, 2019.

18. Takeshita S, Fumoto T, Matsuoka K, Park KA, Aburatani H, Kato S, Ito $\mathrm{M}$ and Ikeda K: Osteoclast-secreted CTHRC1 in the coupling of bone resorption to formation. J Clin Invest 123: 3914-3924, 2013

19. Pyagay P, Heroult M, Wang Q, Lehnert W, Belden J, Liaw L, Friesel RE and Lindner V: Collagen triple helix repeat containing 1, a novel secreted protein in injured and diseased arteries, inhibits collagen expression and promotes cell migration. Circ Res 96: 261-268, 2005.

20. Yang XM, You HY, Li Q, Ma H, Wang YH, Zhang YL, Zhu L, Nie HZ, Qin WX, Zhang ZG, et al: CTHRC1 promotes human colorectal cancer cell proliferation and invasiveness by activating Wnt/PCP signaling. Int J Clin Exp Pathol 8: 12793-12801, 2015.

21. He W, Zhang H, Wang Y, Zhou Y, Luo Y, Cui Y, Jiang N, Jiang W, Wang H, Xu D, et al: CTHRC1 induces non-small cell lung cancer (NSCLC) invasion through upregulating MMP-7/MMP-9. BMC Cancer 18: 400, 2018.

22. Tang L, Dai DL, Su M, Martinka M, Li G andZhou Y: Aberrant expression of collagen triple helix repeat containing 1 in human solid cancers. Clin Cancer Res 12: 3716-3722, 2006.

23. Guo B, Yan H, Li L, Yin K, Ji F and Zhang S: Collagen triple helix repeat containing 1 (CTHRC1) activates Integrin $\beta 3 / F A K$ signaling and promotes metastasis in ovarian cancer. $\mathrm{J}$ Ovarian Res 10: 69, 2017.

24. Tang Z, Wang Y, Liu X and Liu Q: An immunohistochemical study of CTHRC1, Vimentin, E-cadherin expression in papillary thyroid carcinoma. Lin Chung Er Bi Yan Hou Tou Jing Wai Ke Za Zhi 32: 595-598, 2018 (In Chinese).

25. Park EH, Kim S, Jo JY, Kim SJ, Hwang Y, Kim JM, Song SY, Lee DK and Koh SS: Collagen triple helix repeat containing-1 promotes pancreatic cancer progression by regulating migration and adhesion of tumor cells. Carcinogenesis 34: 694-702, 2013.

26. Lee J, Song J, Kwon ES, Jo S, Kang MK, Kim YJ, Hwang Y, Bae H, Kang TH, Chang S, et al: CTHRC1 promotes angiogenesis by recruiting Tie2-expressing monocytes to pancreatic tumors. Exp Mol Med 48: e261, 2016.

27. Li LY, Yin KM, Bai YH, Zhang ZG, Di W and Zhang S: CTHRC1 promotes M2-like macrophage recruitment and myometrial invasion in endometrial carcinoma by integrin-Akt signaling pathway. Clin Exp Metastasis 36: 351-363, 2019.

28. Chandrashekar DS, Bashel B, Balasubramanya SA, Creighton CJ, Ponce-Rodriguez I, Chakravarthi BV and Varambally S: UALCAN: A portal for facilitating tumor subgroup gene expression and survival analyses. Neoplasia 19: 649-658, 2017.

29. Anaya J: OncoLnc: Linking TCGA survival data to mRNAs, miRNAs, and lncRNAs. PeerJ Comput Sci 2: e67, 2016.
30. Chen G, Wang D, Zhao X, Cao J, Zhao Y, Wang F, Bai J, Luo D and Li L: miR-155-5p modulates malignant behaviors of hepatocellular carcinoma by directly targeting CTHRC1 and indirectly regulating GSK-3 $\beta$-involved Wnt $/ \beta$-catenin signaling. Cancer Cell Int 17: 118, 2017.

31. Livak KJ and Schmittgen TD: Analysis of relative gene expression data using real-time quantitative PCR and the 2(-Delta Delta C(T)) method. Methods 25: 402-408, 2001.

32. Saed L, Varse F, Baradaran HR, Moradi Y, Khateri S, Friberg E, Khazaei Z, Gharahjeh S, Tehrani S, Sioofy-Khojine AB, et al: The effect of diabetes on the risk of endometrial Cancer: An updated a systematic review and meta-analysis. BMC Cancer 19: $527,2019$.

33. Nomura H, Aoki D, Michimae H, Mizuno M, Nakai H, Arai M, Sasagawa M, Ushijima K, Sugiyama T, Saito M, et al; Japanese Gynecologic Oncology Group: Effect of taxane plus platinum regimens vs. doxorubicin plus cisplatin as adjuvant chemotherapy for endometrial cancer at a high risk of progression: A Randomized Clinical Trial. JAMA Oncol 5: 833-840, 2019.

34. Durmus T, LeClair RJ, Park KS, Terzic A, Yoon JK and Lindner V: Expression analysis of the novel gene collagen triple helix repeat containing-1 (Cthrc1). Gene Expr Patterns 6: 935-940, 2006

35. Lau EY, Lo J, Cheng BY, Ma MK, Lee JM, Ng JK, Chai S, Lin CH, Tsang SY, Ma S, et al: Cancer-associated fibroblasts regulate tumor-initiating cell plasticity in hepatocellular carcinoma through c-Met/FRA1/HEY1 signaling. Cell Rep 15: $1175-1189,2016$

36. Tan F, Liu F, Liu H, Hu Y, Liu D and Li G: CTHRC1 is associated with peritoneal carcinomatosis in colorectal cancer: A new predictor for prognosis. Med Oncol 30: 473, 2013.

37. Ma MZ, Zhuang C, Yang XM, Zhang ZZ, Ma H, Zhang WM, You H, Qin W, Gu J, Yang S, et al: CTHRC1 acts as a prognostic factor and promotes invasiveness of gastrointestinal stromal tumors by activating Wnt/PCP-Rho signaling. Neoplasia 16: 265-278, 278.e1-278.e13, 2014.

38. Berx G and van Roy F: Involvement of members of the cadherin superfamily in cancer. Cold Spring Harb Perspect Biol 1: a003129, 2009.

39. Cavallaro U and Christofori G: Cell adhesion and signalling by cadherins and Ig-CAMs in cancer. Nat Rev Cancer 4: 118-132, 2004.

40. Wang $\mathrm{P}$, Wang YC, Chen XY, Shen ZY, Cao H, Zhang YJ, Yu J, Zhu JD, Lu YY and Fang JY: CTHRC1 is upregulated by promoter demethylation and transforming growth factor- $\beta 1$ and may be associated with metastasis in human gastric cancer. Cancer Sci 103: 1327-1333, 2012.

41. Liu G, Sengupta PK, Jamal B, Yang HY, Bouchie MP, Lindner V, Varelas $\mathrm{X}$ and Kukuruzinska MA: N-glycosylation induces the CTHRC1 protein and drives oral cancer cell migration. J Biol Chem 288: 20217-20227, 2013.

This work is licensed under a Creative Commons Attribution-NonCommercial-NoDerivatives 4.0 International (CC BY-NC-ND 4.0) License. 